\title{
Formylation, dicyanovinylation and tricyanovinylation of 5-alkoxy- and 5-amino- substituted 2,2'-bithiophenes
}

\author{
M. Manuela M. Raposo*a and G. Kirsch ${ }^{\mathrm{b}}$ \\ a Departamento de Química, Universidade do Minho, Campus de Gualtar \\ 4710-057 Braga, Portugal. \\ b Laboratoire d’Ingénierie Moléculaire et Biochimie Pharmacologique, \\ Université de Metz, Ile de Saulcy, F-57405 Metz Cedex, France.
}

\begin{abstract}
Several donor-acceptor-substituted bithiophenes were synthesized by functionalization of the corresponding 5-alkoxy- or 5-aminobithiophenes $\mathbf{1}$ by different methods: Vilsmeier formylation, metalation followed by reaction with DMF, direct tricyanovinylation reaction using TCNE or Knoevenagel condensation starting from the corresponding 5-formyl- derivatives of $\mathbf{1}$.
\end{abstract}

Keywords: donor-acceptor bithiophene compounds, Vilsmeier formylation, $\alpha$ lithiation, Knoevenagel condensation, tricyanovinylation, non-linear optical material, NLO applications.

\section{Introduction}

For the past few years interest has been focused on new donor-acceptor-substituted thiophene and bithiophene derivatives. Donor-acceptor bithiophene chromophores exibit enhanced second-order polarizabilities $\beta$ compared to biphenyls or stilbenes. The larger nonlinearities were attributed to the bathocromic effect of sulfur, the partial decrease of aromatic character and an increased $\pi$-overlap between the thiophene units. $^{1-9}$ This type of compound can therefore be applied in electro-optical devices. ${ }^{7-12}$ Donor-acceptor-substituted 2,2'-bithiophenes are usually prepared by cross-coupling reactions of electron donor-substituted thiophenes with acceptor-substituted halothiophenes, via organozinc, organotin, or organoboron derivatives. ${ }^{1-6}$

\footnotetext{
* Corresponding author. Phone: +351 253 604381; Fax: +351 253 678983; e-mail: mfox@quimica.uminho.pt
} 
As part of our ongoing effort to develop chromophores for non-linear optical applications ${ }^{13-16}$ we synthesized several donor-acceptor 5,5'-disubstituted 2,2'bithiophenes by functionalization of the corresponding 5-amino- and 5-alkoxybithiophenes 1a-h. ${ }^{17}$ We have recently reported the synthesis of 5-alkoxy- and 5-amino2,2'-bithiophenes which made these compounds available in reasonable amounts, ready for further applications. Indeed, we were able to use these compounds successfully as substrates for the functionalization at the $5^{\prime}$ - position of derivatives 1a-h.

We describe here the synthesis and the reactivity studies of bithiophenes, (formyl, dicyanovinyl and tricyano derivatives), 2 - 6 prepared from 5-alkoxy- and 5-aminobithiophenes 1.

\section{Results and discussion}

Reactivity studies of bithiophenes $\mathbf{1}$ were made through the Vilsmeier-Haack reaction, $\alpha$-lithiation followed by quenching with DMF, Knoevenagel condensation starting from the corresponding 5-formyl- derivatives of $\mathbf{1}$, malononitrile and by direct tricyanovinylation reaction with TCNE.

The formylation of thiophene and oligothiophene derivatives is usually achieved by two methods: through the Vilsmeier reaction, ${ }^{18-21}$ (or by a modified procedure of the Vilsmeier formylation using $\mathrm{DMF} / \mathrm{POCl}_{3}$ in dichloroethane ${ }^{22-24}$ ) or by metalation followed by formyldelithiation using DMF.7, 21, 25-27 Meth-Cohn et al ${ }^{19}$ have recently published a study of the regioselective electrophilic Vilsmeier formylation of 3substituted thiophenes which clearly evidences the effect of the increasing size of the Vilsmeier reagent. They showed that the regioselective Vilsmeier formylation of 3substituted thiophenes may be optimized with either small (obtention of the 2-isomer) or large planar aromatic Vilsmeier reagents (obtention of the 5-isomer).

In our study of the Vilsmeier-Haack formylation of compounds 1f-g with DMF, the ortho position to the alkoxyl or to the 5-N,N-dialkylamino groups of bithiophenes 1 showed to be much more reactive than the 5'-position. Therefore, the Vilsmeier-Haack formylation of 5-alkoxy- and 5- $N, N$-dialkylamino bithiophenes $\mathbf{1 f - g}$, with $\mathrm{DMF} / \mathrm{POCl}_{3}$ at $60{ }^{\circ} \mathrm{C}$ for $2 \mathrm{~h}$., produced a mixture of 4-formyl- derivatives $\mathbf{2 f - g}$ and 4,5'-diformylbithiophenes 3f-g instead of the desired 5-formyl- derivatives (Scheme 1). 
These results showed that in the case of the Vilsmeier formylation of the $5-N, N$ dialkylamino-2,2'-bithiophenes $\mathbf{1 f}-\mathbf{g}$, the reaction occurs in the most activated positions: 4- and $5^{\prime}-$.

\section{$<$ SCHEME 1>}

In both cases, especially for 5-N,N-diisopropylamino-2,2'-bithiophene $\mathbf{1 f}$ the results indicate that even with steric hindrance, the 4-position is still favoured compared to the $5^{\prime}$-position. Monosubstitution at $5^{\prime}$ is never observed. Despite the steric hindrance in position 4-, and given that the formylating agent is not a sterically bulky species, ${ }^{19}$ this position is still the most activated for the electrophilic formylation.

4-Formyl-derivatives $\mathbf{2 f - g}$ were obtained in isolated yields from 41 to $80 \%$ while $4,5^{\prime}$ diformyl-derivatives $\mathbf{3 f - g}$ were isolated in yields from 3 to $10 \%$ (Table 1).

\section{$<$ TABLE 1>}

As $5^{\prime}$-formyl- derivatives 4 could not be synthesized by the Vilsmeier-Haack reaction, we tried to prepare these compounds by lithiation followed by treatment with DMF. The synthesis of $5^{\prime}$-formyl-bithiophenes 4 was therefore achieved by metalation, using $n$-BuLi followed by quenching with DMF, in moderate to good yields.

The metalation was run in $n$-BuLi in dry ether at $0{ }^{\circ} \mathrm{C}$ for $1 \mathrm{~h}$. Subsequently, the organolithium derivatives were converted to the corresponding 5-formyl- compounds $\mathbf{4}$, by addition of DMF followed by refluxing the mixture for 1 to $2.5 \mathrm{~h}$ (Scheme 2).

\section{<SCHEME 2>}

5'-Formyl-2,2'-bithiophenes 4 were obtained in isolated yields from 16 to $88 \%$ (Table 2).

Through this method bithiophenes 1 were selectively lithiated at the $5^{\prime}$-position and subsequently formylated.

\section{$<$ TABLE 2>}


5-Formylbithiophenes $\mathbf{4 a}, \mathbf{d}$ and $\mathbf{g}$ have already been synthesized by other methods such as Pd-catalyzed cross-coupling reactions via zinc-substituted thiophenes ${ }^{3}$ or $v i a$ organotin compounds. ${ }^{4}$

Condensation of aldehydes 4 with malononitrile 28 in refluxing ethanol gave $5^{\prime}-$ dicyanovinyl- derivatives 5 (Scheme 3) in moderate to good yields (45-88\%).

\section{$<$ SCHEME 3>}

As expected, the acceptor strength increase of the dicyanovinyl group in compounds $\mathbf{5}$ induces a bathochromic shift of the $\lambda_{\max }$ in the UV-Vis. spectra (Table 3), as compared to the starting aldehydes $\mathbf{4}$ (Table 2) as well as the mono and dialdehydes $\mathbf{2}$ and $\mathbf{3}$ from the Vilsmeier reaction (Table 1).

\section{$<$ TABLE 3>}

5-Dicyanobithiophenes $\mathbf{5 d}$, e and $\mathbf{g}$ have already been synthesized by other methods, like cross-coupling reactions 3,4 or by reaction of 2,2-dicyanoethenyl-substituted bromoalkanes with 3-aminothioacrylamides. ${ }^{29}$

Three synthetic routes are widely used for the preparation of tricyanovinyl derivatives: direct reaction of tetracyanoethylene (TCNE) with activated aromatic rings, ${ }^{30-31}$ condensation of an aldehyde with malononitrile followed by the reaction with potassium cyanide and oxidation with lead tetraacetate, 30 or lithiation followed by quenching with TCNE. ${ }^{32-33}$ This novel approach to tricyanovinylation in thienyl-imidazoles by reaction of tetracyanoethylene with the thienyllithium derivatives in THF was reported for the first time by $\mathrm{Bu}$ et al $^{32}$.

To continue the reactivity study and to introduce more powerful electrodrawing groups, we used the direct tricyanovinylation reaction in bithiophenes $\mathbf{1}$. The tricyanovinylation of $N, N$-substituted aromatic amines with TCNE occurs with para-substitution, generally in the position of highest electron density. On account of the bulkiness of tricyanovinyl group, the steric factors play a dominant role in determining the course of the reaction of TCNE with 5-N,N-substituted bithiophenes 1. Thus, ortho tricyanovinylation of these compounds does not take place readily. ${ }^{34}$ In the case of the direct tricyanovinylation 
reaction in bithiophenes $\mathbf{1}$, the para position is much more reactive. The tricyanovinylation of bithiophenes 1 therefore occurred exclusively at the 5'-position.

This functionalization was made by reacting the activated 5-alkoxy- and 5-aminobithiophenes 1 with TCNE in DMF for $24 \mathrm{~h}$ at room temperature (Scheme 4).

\section{<SCHEME 4>}

Compounds 6 were obtained in moderate to good yieds (51-87\%) (Table 4).

\section{<TABLE 4>}

5-Tricyanobithiophenes $\mathbf{6 d}, \mathbf{e}$ and $\mathbf{g}$ have previously been described in the literature. Derivatives $\mathbf{6 d}$ and $\mathbf{6 e}$ were prepared by reaction of 1,2,2-tricyanoethenyl-substituted bromoalkanes with 3 -aminothioacrylamides ${ }^{29}$ or by a cross-coupling reaction. ${ }^{5}$

Bithiophene derivatives 4, 5 and 6 exhibit an absorption band in the UV or visible range whose position is strongly influenced by the structure of the compounds, for example by the substitution pattern in the donor and acceptor moieties. ${ }^{29}$ For all of the compounds studied, the tricyanovinyl derivatives absorb at longer wavelength than their formyl- or dicyanovinyl- analogues (Tables 2, 3 and 4). It should be noted that absorption in the visible range is a characteristic feature of all dicyanovinyl- and tricyanovinylsubstituted bithiophenes 5 and $\mathbf{6}$ (Table 3 and 4).

In general, the stronger the donor and/or acceptor group, the smaller the energy difference between ground and excited states, and the longer the wavelenght of absorption. ${ }^{3}$ According to Zyss ${ }^{11}$ the increase of the $\beta$ values characteristics of the NLO effects are accompanied by an increase of the $\lambda_{\max }$ in the UV-Vis. spectra.

Bithiophene derivatives $\mathbf{2}$ - $\mathbf{6}$ were completely characterized by elemental analysis and/or HRMS, ${ }^{1} \mathrm{H}$ and ${ }^{13} \mathrm{C}$ spectroscopy, IR and UV-Vis. spectroscopy (Tables 1-4). The non-linear optical properties of the new push-pull systems $\mathbf{2 f - g , ~ 3 f - g , ~} \mathbf{4 b}-\mathbf{c}, \mathbf{4 e - f}, \mathbf{4 h}$, 5a-b, 5f, 6a-b, $6 \mathbf{f}$ will be investigated in the future. 


\section{Conclusions}

Starting from the easily available 5-alkoxy- and 5-amino-2,2'-bithiophenes 1, commercial reagents as well as simple and convenient procedures were used to synthesize several formyl-, dicyanovinyl- and tricyanovinyl- 2,2'-bithiophenes in moderate to good yields, via four methods: i) Vilsmeier formylation, ii) lithiation followed by reaction with DMF, iii) Knoevenagel condensation of the corresponding formyl derivatives with malononitrile and iv) direct tricyanovinylation reaction with TCNE.

In agreement with previous findings $3-5,29,35$ the new compounds prepared can be applied for the manufacture of new materials with strong non-linear optical (NLO) properties.

\section{Experimental}

${ }^{1} \mathrm{H}$ NMR spectra were obtained on a Varian Unity Plus Spectrometer at $300 \mathrm{MHz}$ and ${ }^{13} \mathrm{C}$ NMR spectra were determined on a Varian Unity Plus Spectrometer at $75.4 \mathrm{MHz}$ using the solvent peak as internal reference. The solvents are quoted in parentheses before the chemical shift values ( $\delta$ relative to TMS). Melting points were determined on a Gallenkamp apparatus and are uncorrected. Infrared spectra were recorded on a Perkin Elmer 1600 FTIR spectrophotometer. UV spectra were recorded in ethanol on a Hitachi U-2000. EI mass spectra EI (70 eV) and HRMS were run on a Unicam GC-MS 120. Elemental analyses were made on a Leco CHNS-932. Column chromatography was performed on Merck silica gel 60 (Art 9385). Light petroleum refers to solvent boiling in the range $40-60^{\circ} \mathrm{C}$.

The synthesis of bithiophenes $\mathbf{1 a - h}$ has been described elsewhere. ${ }^{17}$

General procedure for the synthesis of 4-formyl-2,2'-bithiophenes $2 \mathrm{f}$-g and 4,5'diformyl-2,2'-bithiophenes 3f-g from bithiophenes 1f-g through Vilsmeyer formylation

$\mathrm{POCl}_{3}(4.8 \mathrm{mmol})$ was added to DMF $(4.8 \mathrm{mmol})$ at $0{ }^{\circ} \mathrm{C}$ and the mixture was stirred for $15 \mathrm{~min}$. at $0^{\circ} \mathrm{C}$. After this time bithiophenes $\mathbf{1 f}-\mathbf{g}$ (4.0 mmol) dissolved in DMF (2 
$\mathrm{ml}$ ) were added dropwise with stirring. The reaction mixture was then heated $2 \mathrm{~h}$ at 60 ${ }^{\circ} \mathrm{C}$. The solution was then poured slowly into $75 \mathrm{ml}$ saturated sodium acetate aqueous solution and stirred $30 \mathrm{~min}$. The organic layer was diluted with ether, washed with saturated $\mathrm{NaHCO}_{3}$ aqueous solution, and dried with anhydrous $\mathrm{Na}_{2} \mathrm{SO}_{4}$. Evaporation of the organic extract under reduced pressure gave a mixture of 4-formyl- $\mathbf{2 f - g}$ and 4,5'diformyl-bithiophenes $\mathbf{3 f - g}$ which were purified by "flash" chromatography on silica with increasing amounts of ether in light petroleum as eluent.

Vilsmeyer formylation of $\mathbf{1 f}$ gave a mixture of 4-formyl-5- $N, N$-diisopropylamino-2,2'bithiophene $2 \mathbf{f}$ and 4,5'-diformyl-5- $N, N$-diisopropylamino-2, $2^{\prime}$-bithiophene 3f. The first component eluted was 4-formyl-5-N,N-diisopropylamino-2,2'-bithiophene $\mathbf{2 f}$ as a pale yellow solid (41\%). Mp: 56.5-57.5 ${ }^{\circ} \mathrm{C}$. UV (EtOH): $\lambda_{\max } \mathrm{nm}\left(\varepsilon, / \mathrm{M}^{-1} \mathrm{~cm}^{-1}\right), 313.0$ (10878), 257.0 (14330). IR (KBr): v 3120, 3080, 2980, 1668 (CHO), 1560, 1520, 1480, 1460, 1440, 1390, 1340, 1230, 1220, 1170, 1125, 1100, 1080, 1040, 1000, 900, 825, 745, 740, 720, $700 \mathrm{~cm}^{-1} .{ }^{1} \mathrm{H} \mathrm{NMR}\left(\mathrm{CDCl}_{3}\right) \delta 1.14\left(\mathrm{~d}, 12 \mathrm{H}, J=6.5 \mathrm{~Hz}, 2 \times \mathrm{CH}\left(\mathrm{CH}_{3}\right)_{2}\right)$, 3.45-3.65 (m, 2 H, 2xCH( $\left.\left.\mathrm{CH}_{3}\right)_{2}\right)$, 6.99-7.03 (m, $\left.1 \mathrm{H}, 4^{\prime}-\mathrm{H}\right)$, 7.16-7.17 (m, $\left.1 \mathrm{H}, 3^{\prime}-\mathrm{H}\right)$, 7.22-7.26 (m, $\left.1 \mathrm{H}, 5^{\prime}-\mathrm{H}\right), 7.35$ (s, 1H, 3-H), 9.92 (s, 1H, CHO). ${ }^{13} \mathrm{C} \mathrm{NMR}\left(\mathrm{CDCl}_{3}\right) \delta$ 21.18, 52.09, 118.06, 124.01, 124.88, 127.75, 132.84, 137.07, 139.02, 163.28, 185.82. MS (EI) m/z (\%): 293 (M+, 100), 278 (17), 264 (9), 250 (52), 232 (33), 208 (39), 192 (5), 181 (15), 153 (9), 121 (12), 96 (10), 69 (6). Anal. Calcd for $\mathrm{C}_{15} \mathrm{H}_{19} \mathrm{NOS}_{2}$ : C, 61.42; H, 6.48; N, 4.77; S, 21.86. Found: C, 61.39; H, 6.47; N, 4.77; S, 21.73. The second component eluted was $4,5^{\prime}$-diformyl-5- $N, N$-diisopropylamino-2,2'-bithiophene $\mathbf{3 f}$ as an orange oil (10\%). UV (EtOH): $\lambda$ max $\mathrm{nm}\left(\varepsilon, / \mathrm{M}^{-1} \mathrm{~cm}^{-1}\right), 343.0$ (18039), 241.5 (4914), 214.0 (6102). IR (liquid film): v2973, 2932, 2872, 1665 broad (CHO), 1547, 1485, 1449, 1380, 1115, 1050, 811, 751, $664 \mathrm{~cm}^{-1} .{ }^{1} \mathrm{H} \mathrm{NMR}\left(\mathrm{CDCl}_{3}\right) \delta 1.18(\mathrm{~d}, 12 \mathrm{H}, J=6.5$ $\left.\mathrm{Hz} 2 \mathrm{xCH}\left(\mathrm{CH}_{3}\right)_{2}\right), 3.58-3.79\left(\mathrm{~m}, 2 \mathrm{H}, 2 \mathrm{xCH}\left(\mathrm{CH}_{3}\right)_{2}\right), 7.22$ (d, $\left.1 \mathrm{H}, J=4.0 \mathrm{~Hz}, 3^{\prime}-\mathrm{H}\right), 7.53$ (s, $1 \mathrm{H}, 3-\mathrm{H}), 7.66$ (d, $\left.1 \mathrm{H}, J=4.0 \mathrm{~Hz}, 4^{\prime}-\mathrm{H}\right), 9.85$ (s, $\left.1 \mathrm{H}, \mathrm{CHO}\right), 9.89$ (s, $\left.1 \mathrm{H}, \mathrm{CHO}\right)$. ${ }^{13} \mathrm{C} \mathrm{NMR}\left(\mathrm{CDCl}_{3}\right) \delta$ 21.22, 52.71, 121.63, 124.22, 129.94, 137.18, 137.30, 141.83, 146.68, 165.11, 182.47, 185.08. MS (EI) $m / z$ (\%): $321\left(\mathrm{M}^{+}, 100\right), 306$ (15), 278 (64), 264 (30), 260 (14), 246 (8), 237 (30), 208 (6), 186 (13), 155 (9), 146 (6), 121 (7), 111 (13), 96 (9). HRMS: $m / z$ (EI) for $\mathrm{C}_{16} \mathrm{H}_{19} \mathrm{NO}_{2} \mathrm{~S}_{2}$; calcd 321.0857; found: 321.0856.

Vilsmeyer formylation of $\mathbf{1 g}$ gave a mixture of 4-formyl-5-piperidino-2,2'-bithiophene $\mathbf{2 g}$ and 4,5'-diformyl-5-piperidino-2,2'-bithiophene $3 \mathrm{~g}$. The first component eluted was 
4-formyl-5-piperidino-2, $2^{\prime}$-bithiophene $2 \mathrm{~g}$ as a beige solid (80\%). Mp: $71-72{ }^{\circ} \mathrm{C}(n-$ hexano). UV (EtOH): $\lambda_{\max } \mathrm{nm}\left(\varepsilon, / \mathrm{M}^{-1} \mathrm{~cm}^{-1}\right) 332.0$ (14794), 253.0 (17719), 214.0 (6102). IR (liquid film): $v$ 2936, 2920, 1660 (CHO), 1562, 1511, 1495, 1461, 1442, 1381, 1333, 1246, 1165, 1127, 1074, 1040, 991, 910, 826, 752, $694 \mathrm{~cm}^{-1} .{ }_{1}^{1} \mathrm{H}$ NMR $\left(\mathrm{CDCl}_{3}\right) \delta$ 1.60-1.70 (m, $\left.2 \mathrm{H}, \mathrm{CH}_{2}\right), 1.70-1.90(\mathrm{~m}, 4 \mathrm{H}, 2 \mathrm{xCH} 2), 3.30-3.40(\mathrm{~m}, 4 \mathrm{H}$, 2xCH$\left.{ }_{2}\right), 6.98-7.06\left(\mathrm{~m}, 1 \mathrm{H}, 4^{\prime}-\mathrm{H}\right), 7.17$ (dd, $1 \mathrm{H}, J=3.6$ and $\left.1.2 \mathrm{~Hz}, 3^{\prime}-\mathrm{H}\right), 7.30(\mathrm{~s}, 1 \mathrm{H}$, $3-\mathrm{H}), 7.39$ (dd, $1 \mathrm{H}, J=5.1$ and $\left.1.2 \mathrm{~Hz}, 5^{\prime}-\mathrm{H}\right), 9.83$ (s, $\left.1 \mathrm{H}, \mathrm{CHO}\right) .{ }^{13} \mathrm{C} \mathrm{NMR}\left(\mathrm{CDCl}_{3}\right)$ $\delta$ 23.55, 25.43, 56.25, 122.66, 123.00, 123.94 (2 overlapped signals), 124.18, 127.64, 136.97, 168.56, 182.57. MS (EI) $\mathrm{m} / z$ (\%): 277 (M+, 100), 260 (89), 227 (6), 194 (11), 178 (6), 127 (9), 122 (9), 69 (5). Anal. Calcd for $\mathrm{C}_{14} \mathrm{H}_{15} \mathrm{NOS}_{2}$ : C, 60.64; H, 5.41; N, 5.05; S, 23.13. Found: C, 60.45; H, 5.47; N, 5.11; S, 22.80. The second component eluted was $4,5^{\prime}$-diformyl-5-piperidino-2, $2^{\prime}$-bithiophene $\mathbf{3} \mathrm{g}$ as a pale orange solid (3\%). Mp: 113.5-115 ${ }^{\circ} \mathrm{C}$. UV (EtOH): $\lambda_{\max } \mathrm{nm}\left(\varepsilon, / \mathrm{M}^{-1} \mathrm{~cm}^{-1}\right) 397.0$ (14735), 248.0 (12235), 213.0 (5928). IR (liquid film): $v 1659$ (CHO), 1650 (CHO), 1546, 1509, 1444, 1382, 1226, 1163, 1129, 1050, 1020, 991, 852, $795 \mathrm{~cm}^{-1} .{ }^{1} \mathrm{H}$ NMR $\left(\mathrm{CDCl}_{3}\right) \delta 1.50-1.70(\mathrm{~m}, 2$ $\left.\mathrm{H}, \mathrm{CH}_{2}\right), 1.75-1.85\left(\mathrm{~m}, 4 \mathrm{H}, 2 \mathrm{xCH}_{2}\right) 3.95-4.43\left(\mathrm{~m}, 4 \mathrm{H}, 2 \mathrm{xCH}_{2}\right), 7.08(\mathrm{~d}, 1 \mathrm{H}, J=4.0$ Hz, 3'-H), 7.46 (s, 1 H, 3-H), 7.64 (d, 1 H, J=4.0 Hz, 4'-H), 9.78 (s, 1 H, CHO), 9.83 (s, $1 \mathrm{H}, \mathrm{CHO}) .{ }^{13} \mathrm{C}$ NMR $\left(\mathrm{CDCl}_{3}\right) \delta$ 23.46, 25.34, 55.92, 121.16, 122.91, 123.07, 126.92, 137.46, 140.79, 147.04, 168.92, 182.21 (2 overlapped signals). MS (EI) $\mathrm{m} / \mathrm{z}$ (\%): 305 $\left(\mathrm{M}^{+}, 100\right), 288$ (81), 277 (10), 260 (10), 222 (5), 170 (8), 149 (10), 121 (6), 111 (15), 97 (9), 83 (50). HRMS: $m / z$ (EI) for $\mathrm{C}_{15} \mathrm{H}_{15} \mathrm{NO}_{2} \mathrm{~S}_{2}$; calcd 305.0544; found: 305.0548.

General procedure for the synthesis of 5-formyl-2,2'-bithiophenes 4 from 2,2'bithiophenes 1 via metalation with $\boldsymbol{n}$-BuLi followed by reaction with DMF

A $2.5 \mathrm{M}$ solution of $n$-BuLi in hexanes $(1.6 \mathrm{ml}, 4.0 \mathrm{mmol})$ was dropped under Ar at $0^{\circ}$ $\mathrm{C}$ to a stirred solution of bithiophenes $\mathbf{1}$ in anhydrous ether $(2.0 \mathrm{mmol})$. The reaction mixture was then stirred $1 \mathrm{~h}$ at $0^{\circ} \mathrm{C}$ and was allowed to stand $15 \mathrm{~min}$. at room temperature. DMF (0.18 g, $2.4 \mathrm{mmol})$ dissolved in anhydrous ether $(2 \mathrm{ml})$ was added dropwise at r.t. The mixture was heated at reflux for 1-2.5 h. The mixture was poured into water $(20 \mathrm{ml})$ and extracted with $(3 \times 50 \mathrm{ml})$ of ethyl acetate. The combined organic extracts were washed with $\mathrm{H}_{2} \mathrm{O}(100 \mathrm{ml})$, dried with $\mathrm{Na}_{2} \mathrm{SO}_{4}$ and the solvent was evaporated under reduced pressure to give the crude 5-formyl-2,2'-bithiophenes 4 
which were purified by "flash" chromatography on silica with increasing amounts of ether in light petroleum as eluent.

5-Formyl-5'-methoxy-2,2'-bithiophene $4 \mathbf{a}^{3}$ : orange solid $(45 \%)$. Mp: $56-58{ }^{\circ} \mathrm{C}$. Recristalization from $n$-hexane gave a pale orange solid mp 61-62 ${ }^{\circ} \mathrm{C}$ [lit. ${ }^{3} 55-57{ }^{\circ} \mathrm{C}$ ]. UV (EtOH): $\lambda_{\max } \mathrm{nm}\left(\varepsilon, / \mathrm{M}^{-1} \mathrm{~cm}^{-1}\right) 385$ (22351), 379 (24319), 254 inf. (3511). IR (liquid film): $v 1646$ (CHO), 1480, 1466, 1443, 1422, 1382, 1248, 1231, 1156, 1061, 1045, 989, 878, 795, $770 \mathrm{~cm}^{-1} .{ }^{1} \mathrm{H}$ NMR $\left(\mathrm{CDCl}_{3}\right) \delta 3.96$ (s, $\left.3 \mathrm{H}, \mathrm{OCH}_{3}\right) 6.19(\mathrm{~d}, 2 \mathrm{H}$, $\left.J=4.0 \mathrm{~Hz}, 4^{\prime}-\mathrm{H}\right), 7.04-7.08$ (m, 2 H, 3 and 3'-H), 7.63 (d, 1 H, $\left.J=4.0 \mathrm{~Hz}, 4-\mathrm{H}\right), 9.82$ (s, 1 $\mathrm{H}, \mathrm{CHO}) .{ }^{13} \mathrm{C} \mathrm{NMR} \delta\left(\mathrm{CDCl}_{3}\right) \delta 60.36,105.12,121.33,122.38,124.58,137.56$, 140.33, 148.27, 168.01, 182.22. MS (EI) m/z (\%): $224\left(\mathrm{M}^{+}, 100\right), 209$ (88), 193 (5), 181 (18), 153 (18), 137 (20), 109 (7), 69 (14). Anal. Calcd for $\mathrm{C}_{10} \mathrm{H}_{8} \mathrm{O}_{2} \mathrm{~S}_{2}$ : C, 53.56; $\mathrm{H}$, 3.57; S, 28.60. Found: C, 53.44; H, 3.65; S, 28.52.

5-Formyl-5'-ethoxy-2,2'-bithiophene 4b : orange solid (56\%). Mp: 62-64 ${ }^{\circ} \mathrm{C}$. Recristalization from $n$-hexane gave a orange solid mp 66.5-68.5 ${ }^{\circ} \mathrm{C}$. UV (EtOH): $\lambda_{\max }$ $\mathrm{nm}\left(\varepsilon, / \mathrm{M}^{-1} \mathrm{~cm}^{-1}\right) 383.0$ (15579), 257.0 (6921). IR (KBr) v 2980, 2940, 1660 (CHO), 1520, 1480, 1460, 1440, 1380, 1360, 1250, 1230, 1200, 1110, 1040, 870, 800, $760 \mathrm{~cm}^{-1}$. ${ }^{1} \mathrm{H} \mathrm{NMR}\left(\mathrm{CDCl}_{3}\right) \delta 1.45\left(\mathrm{t}, 3 \mathrm{H}, J=7.0 \mathrm{~Hz}, \mathrm{OCH}_{2} \mathrm{CH}_{3}\right), 4.15(\mathrm{q}, 2 \mathrm{H} \mathrm{J}=7.0 \mathrm{~Hz}$, $\left.\mathrm{OCH}_{2} \mathrm{CH}_{3}\right), 6.18$ (d, $\left.1 \mathrm{H}, J=4.0 \mathrm{~Hz}, 4^{\prime}-\mathrm{H}\right), 7.00-7.10$ (m, $2 \mathrm{H}, 3$ and $\left.3^{\prime}-\mathrm{H}\right), 7.62$ (d, $1 \mathrm{H}$, $J=4.1 \mathrm{~Hz}, 4-\mathrm{H}), 9.81$ (s, $1 \mathrm{H}, \mathrm{CHO}) .{ }^{13} \mathrm{H} \mathrm{NMR}\left(\mathrm{CDCl}_{3}\right) \delta 14.7,69.4,105.4,121.3$, 122.2, 123.1, 123.7, 127.5, 138.1, 164.4. MS (EI) m/z (\%): $238\left(\mathrm{M}^{+}, 74\right), 209$ (100), 153 (22), 149 (24), 137 (45), 127 (13), 121 (11), 109 (23), 97 (6), 93 (12), 82 (18). HRMS: $m / z$ (EI) for $\mathrm{C}_{11} \mathrm{H}_{10} \mathrm{O}_{2} \mathrm{~S}_{2}$; calcd 238.0122; found: 238.0134 .

5-Formyl-5'-isopropyloxy-2,2'-bithiophene 4c : orange solid (27\%). Mp: 71.9-72.3 ${ }^{\circ} \mathrm{C}$. UV (EtOH): $\lambda_{\max } \mathrm{nm}\left(\varepsilon, / \mathrm{M}^{-1} \mathrm{~cm}^{-1}\right) 386.5$ (17133), 254.0 (8079). IR (Nujol) v 2980, 2940, 1652 (CHO), 1485, 1249, 1226, 1104, 1047, 922, 877, 826, 798, 765, $667 \mathrm{~cm}^{-1}$. ${ }^{1} \mathrm{H} \mathrm{NMR}\left(\mathrm{CDCl}_{3}\right) \delta 1.41\left(\mathrm{~d}, 6 \mathrm{H}, J=6.0 \mathrm{~Hz}, \mathrm{OCH}\left(\mathrm{CH}_{3}\right)_{2}\right), 4.25$ (sep, $1 \mathrm{H}, J=6.0 \mathrm{~Hz}$, $\left.\mathrm{OCH}\left(\mathrm{CH}_{3}\right)_{2}\right), 6.21\left(\mathrm{~d}, 1 \mathrm{H}, J=3.9 \mathrm{~Hz}, 4^{\prime}-\mathrm{H}\right), 7.02-7.06$ (m, $2 \mathrm{H}, 3$ and $\left.3^{\prime}-\mathrm{H}\right), 7.63$ (d, 1 $\mathrm{H}, J=3.6 \mathrm{~Hz}, 4-\mathrm{H}$ ), 9.82 (s, $1 \mathrm{H}, \mathrm{CHO}$ ). ${ }^{13} \mathrm{H}$ NMR (Acetone-D 6 ) $\delta 21.94,78.50,108.66$, 122.99, 123.59, 125.79, 139.17, 141.50, 148.05, 166.79, 183.25. MS (EI) $\mathrm{m} / \mathrm{z}$ (\%): 252 $\left(\mathrm{M}^{+}, 11\right), 210$ (100), 162 (7), 151 (6), 137 (7), 113 (7). HRMS: $m / z$ (EI) for $\mathrm{C}_{12} \mathrm{H}_{12} \mathrm{O}_{2} \mathrm{~S}_{2}$; calcd 252.027873; found: 252.028214 . 
5-Formyl-5'-N,N-dimethylamino-2,2'-bithiophene $4 \mathbf{d}^{3}$ : orange solid (86\%). Mp: $114-$ $115{ }^{\circ} \mathrm{C}$. Recristalization from $n$-hexane gave an orange solid mp 116-117.5 ${ }^{\circ} \mathrm{C}$ [lit. ${ }^{3}$ 114-115 ${ }^{\circ} \mathrm{C}$ ]. UV (EtOH): $\lambda_{\max } \mathrm{nm}\left(\varepsilon, / \mathrm{M}^{-1} \mathrm{~cm}^{-1}\right) 451.0$ (25114), 287.0 inf. (3809), 268 (6276), 217.0 (6486). IR (KBr) v 2940, 2880, 2800, 1645 (CHO), 1560, 1530, $1500,1450,1430,1420,1300,1240,1050,920,870,800,760,700,665 \mathrm{~cm}^{-1} .{ }^{1} \mathrm{H}$ NMR $\left(\mathrm{CDCl}_{3}\right) \delta 3.00\left(\mathrm{~s}, 6 \mathrm{H}, \mathrm{N}\left(\mathrm{CH}_{3}\right)_{2}\right), 5.82\left(\mathrm{~d}, 1 \mathrm{H} \mathrm{J}=4.1 \mathrm{~Hz}, 4^{\prime}-\mathrm{H}\right), 6.95$ (d, $1 \mathrm{H} \mathrm{J}=4.1 \mathrm{~Hz}$, $\left.3^{\prime}-\mathrm{H}\right), 7.13$ (d, $\left.1 \mathrm{H}, J=4.1 \mathrm{~Hz}, 3-\mathrm{H}\right) 7.58$ (d, $\left.1 \mathrm{H}, J=4.1 \mathrm{~Hz}, 4-\mathrm{H}\right), 9.76$ (s, 1 H, CHO). ${ }^{13} \mathrm{C} \mathrm{NMR}\left(\mathrm{CDCl}_{3}\right) \delta 42.36,102.73,119.29,120.48,127.61,138.12,138.40,149.62$, 161.08, 181.82. MS (EI) $m / z(\%): 238\left(\mathrm{M}^{+}, 74\right) 237\left(\mathrm{M}^{+}, 100\right), 222(34), 193$ (4), 181 (11), 164 (6), 137 (11), 119 (5), 105 (7), 69 (7). Anal. Calcd for $\mathrm{C}_{11} \mathrm{H}_{11} \mathrm{NOS}_{2}$ : C, 55.69; H, 4.64; N, 5.90; S, 27.03. Found: C, 55.92; H, 4.70; N, 5.92; S, 26.89.

5-Formyl-5'- $N, N$-diethylamino-2,2'-bithiophene 4e: red solid (86\%). Mp: 84-86 ${ }^{\circ} \mathrm{C}$. Recristalization from $n$-hexane gave a red solid mp $90-91^{\circ} \mathrm{C}$. UV (EtOH): $\lambda_{\max } \mathrm{nm}(\varepsilon$, $/ \mathrm{M}^{-1} \mathrm{~cm}^{-1}$ ) 463.0, (27107), 287.0 inf. (4126), 268.0 (5619), 217.0 (5126). IR (KBr) $v$ 3060, 2980, 2940, 2880, 1650 (CHO), 1560, 1520, 1500, 1440, 1380, 1360, 1260, $1240,1180,1140,1100,1070,1060,1000,890,870,860,780,750,690,660 \mathrm{~cm}^{-1} .{ }^{1} \mathrm{H}$ NMR $\left(\mathrm{CDCl}_{3}\right) \delta 1.24\left(\mathrm{t}, 6 \mathrm{H}, J=7.1 \mathrm{~Hz}, 2 \mathrm{xCH}_{2} \mathrm{CH}_{3}\right), 3.30(\mathrm{q}, 4 \mathrm{H}, J=7.1 \mathrm{~Hz}$, 2xCH $\mathrm{CH}_{3}$ ), 5.79 (d, $\left.1 \mathrm{H}, J=4.1 \mathrm{~Hz}, 4^{\prime}-\mathrm{H}\right), 6.92$ (d, $\left.1 \mathrm{H}, J=4.1 \mathrm{~Hz}, 3^{\prime}-\mathrm{H}\right), 7.12$ (d, $1 \mathrm{H}$, $J=4.2 \mathrm{~Hz}, 3-\mathrm{H}), 7.56$ (d, $1 \mathrm{H}, J=4.2 \mathrm{~Hz}, 4-\mathrm{H}), 9.75$ (s, $1 \mathrm{H}, \mathrm{CHO}) .{ }^{13} \mathrm{C} \mathrm{NMR}\left(\mathrm{CDCl}_{3}\right)$ $\delta 12.20,47.15,101.75,117.64,120.08,121.68,127.85,137.99,138.22$, 149.89, 159.21, 181.71. MS (EI) m/z (\%): 265 (M+, 100), 250 (85), 236 (9), 221 (21), (208 (9), 194 (6), 181 (3), 149 (5), 121 (5), 96 (18), 69 (5). Anal. Calcd for $\mathrm{C}_{13} \mathrm{H}_{15} \mathrm{NOS}_{2}$ : C, 58.86; H, 5.65; N, 5.28; S, 24.18. Found: C, 58.80; H, 5.66; N, 5.28; S, 23.87.

5-Formyl-5'- $N, N$-diisopropylamino-2,2'-bithiophene 4f: dark red solid (88\%). Mp: 98$100{ }^{\circ} \mathrm{C}$. Recristalization from $n$-hexane gave a dark red solid mp 100-102 ${ }^{\circ} \mathrm{C}$. UV (EtOH): $\lambda_{\max } \mathrm{nm}\left(\varepsilon, / \mathrm{M}^{-1} \mathrm{~cm}^{-1}\right) 466.0$ (22517), $290.5 \mathrm{inf} .(4386), 270.0$ (5307), 212.0 (5096). IR (KBr) v2970, 2930, 2880, 1650 (CHO), 1540, 1510, 1480, 1440, 1370, 1350, 1060, 1240, 1190, 1160, 1140, 1120, 1070, 1050, 800, 780, 760, 750, 680, 660 $\mathrm{cm}^{-1} .{ }^{1} \mathrm{H} \mathrm{NMR}\left(\mathrm{CDCl}_{3}\right) \delta 1.29\left(\mathrm{~d}, 12 \mathrm{H}, J=6.7 \mathrm{~Hz}, 2 \times \mathrm{CH}\left(\mathrm{CH}_{3}\right)_{2}\right), 3.76$ (sep, $2 \mathrm{H}, J=6.7$ $\left.\mathrm{Hz}, 2 \mathrm{xCH}\left(\mathrm{CH}_{3}\right)_{2}\right), 6.00$ (d, $\left.1 \mathrm{H}, J=3.8 \mathrm{~Hz}, 4^{\prime}-\mathrm{H}\right), 6.94$ (d, $\left.1 \mathrm{H}, J=3.8 \mathrm{~Hz}, 3^{\prime}-\mathrm{H}\right), 7.11$ (1 $\mathrm{H}, \mathrm{d}, J=3.8 \mathrm{~Hz}, 3-\mathrm{H}), 7.57(1 \mathrm{H}, \mathrm{d}, J=3.8 \mathrm{~Hz}, 4-\mathrm{H}), 9.74(\mathrm{~s}, 1 \mathrm{H}, \mathrm{CHO}) .{ }^{13} \mathrm{C}$ NMR 
$\left(\mathrm{CDCl}_{3}\right) \delta 20.36,51.22,106.97,120.43,126.81,138.14,138.30,149.72,157.12$, 181.79. MS (EI) m/z (\%): $293\left(\mathrm{M}^{+}, 100\right), 278$ (45), 250 (20), 236 (55), 220 (3), 208 (71), 180 (4), 149 (6), 121 (4), 96 (21), 69 (4). Anal. Calcd for $\mathrm{C}_{15} \mathrm{H}_{19} \mathrm{NOS}_{2}$ : C, 61.42; H, 6.48; N, 4.77; S, 21.86. Found: C, 61.60; H, 6.79; N, 4.81; S, 21.79.

5-Formyl-5'-piperidino-2,2'-bithiophene $\mathbf{4 g}^{4}$ : orange solid (84\%). Mp: $145-146{ }^{\circ} \mathrm{C}$. Recristalization from $n$-hexane/dichloromethane gave an orange solid $\mathrm{mp}$ 148.5-150 ${ }^{\circ} \mathrm{C}$. [lit. ${ }^{4}$ 143-145 ${ }^{\circ} \mathrm{C}$ ]. UV (EtOH): $\lambda_{\max } \mathrm{nm}\left(\varepsilon, / \mathrm{M}^{-1} \mathrm{~cm}^{-1}\right) 442.0$ (22133), 287.0 inf. (3809), 268.0 (5790), 217.0 (5095). IR (KBr) v 2950, 2840, 2780, 1650 (CHO), 1507, 1490, 1420, 1380, 1250, 1230, 1120, 1080, 1050, 1000, 900, 860, 820, 800, 760, 750, $660 \mathrm{~cm}^{-1} .{ }^{1} \mathrm{H} \mathrm{NMR}\left(\mathrm{CDCl}_{3}\right) \delta 1.50-1.85\left(\mathrm{~m}, 6 \mathrm{H}, 3 \mathrm{xCH} \mathrm{H}_{2}\right), 3.15-3.30\left(\mathrm{~m}, 4 \mathrm{H}, 2 \mathrm{xCH}_{2}\right)$, 6.00 (d, $\left.1 \mathrm{H}, J=4.0 \mathrm{~Hz}, 4^{\prime}-\mathrm{H}\right), 6.97$ (d, $\left.1 \mathrm{H}, J=4.0 \mathrm{~Hz}, 3^{\prime}-\mathrm{H}\right), 7.12$ (d, $1 \mathrm{H}, J=4.0 \mathrm{~Hz}, 3-$ $\mathrm{H}), 7.59$ (d, $1 \mathrm{H}, J=4.0 \mathrm{~Hz}, 4-\mathrm{H}), 9.77$ (s, $1 \mathrm{H}, \mathrm{CHO}) .{ }^{13} \mathrm{C} \mathrm{NMR}\left(\mathrm{CDCl}_{3}\right) \delta 23.60$, 25.02, 51.65, 104.47, 120.25, 120.90, 127.05, 138.02, 138.84, 149.44, 161.74, 181.94. MS (EI) m/z (\%): $277\left(\mathrm{M}^{+}, 100\right), 262$ (3), 236 (3), 221 (10), 207 (7), 192 (3), 149 (3), 121 (3), 96 (5), 69 (3). Anal. Calcd for $\mathrm{C}_{14} \mathrm{H}_{15} \mathrm{NOS}_{2}$ : C, 60.64; H, 5.41; N, 5.05; S, 23.13. Found: C, 60.75; H, 5.47; N, 5.07; S, 23.12.

5-Formyl-5'-(4-methoxyanilino)-2,2'-bithiophene 4h: dark orange solid (16\%). Mp: 122-124 ${ }^{\circ} \mathrm{C}$ (ether/petrol). UV (EtOH): $\lambda$ max $\mathrm{nm}\left(\varepsilon, / \mathrm{M}^{-1} \mathrm{~cm}^{-1}\right) 462.0$ (18895), 313.0 (8987). IR (KBr) v $3320(\mathrm{NH}), 1630(\mathrm{CHO}), 1540,1510,1470,1430,1380,1240$, $1180,1160,1050,830,800,760,660,630 \mathrm{~cm}^{-1} .{ }^{1} \mathrm{H} \quad \mathrm{NMR}\left(\mathrm{CDCl}_{3}\right) \delta 3.81(\mathrm{~s}, 3 \mathrm{H}$ $\left.\mathrm{OCH}_{3}\right), 5.90\left(\mathrm{~d}, 1 \mathrm{H}, J=3.9 \mathrm{~Hz}, 4^{\prime}-\mathrm{H}\right), 6.42\left(\mathrm{~d}, 1 \mathrm{H}, J=3.9 \mathrm{~Hz}, 3^{\prime}-\mathrm{H}\right) 6.88$ (d, $2 \mathrm{H}, J=9.3$ $\mathrm{Hz}, 2 \mathrm{xAr}-H)$, 7.04-7.09 (m, $3 \mathrm{H}, 2 \mathrm{xAr}-H+\mathrm{N} H), 7.13$ (d, $1 \mathrm{H}, J=4.2 \mathrm{~Hz}, 3-\mathrm{H}), 7.61$ (d, $1 \mathrm{H}, J=4.2 \mathrm{~Hz}, 4-\mathrm{H}), 9.80$ (s, $1 \mathrm{H}, \mathrm{CHO}) . \mathrm{MS}(\mathrm{EI}) \mathrm{m} / z(\%): 316\left(\mathrm{M}^{+}+1,20\right), 315$ (M+1, 100), 314 (13), 300 (34), 158, (5), 148 (5), 122 (3), 108 (3), 98 (3). HRMS: m/z (EI) for $\mathrm{C}_{16} \mathrm{H}_{13} \mathrm{NO}_{2} \mathrm{~S}_{2}$; calcd 315.0388; found: 315.0393 .

General procedure for the synthesis of 5-dicyanovinyl-2,2'-bithiophenes 5 from the corresponding 5-formyl-2,2’-bithiophenes 4 by Knoevenagel condensation

To a solution of malononitrile $(0.2 \mathrm{~g}, 3.0 \mathrm{mmol})$ and 5-formyl-bithiophenes 4 (2.5 mmol) in ethanol $(50 \mathrm{ml})$ was added piperidine (1 drop). The solution was heated at reflux during different reaction times (15 min.-3 h), then cooled and the solvent was 
removed under reduced pressure to give the crude 5-dicyanovinyl-2,2'-bithiophenes 5 which were purified by "flash" chromatography on silica with increasing amounts of ether in light petroleum as eluent.

5-Dicyanovinyl-5'-methoxy-2,2'-bithiophene 5a: dark orange solid (46\%). Mp: 154$156{ }^{\circ} \mathrm{C}$. Recristalization from $n$-hexane/toluene gave a dark orange solid mp 166-168 ${ }^{\circ} \mathrm{C}$. UV (EtOH): $\lambda_{\max } \mathrm{nm}\left(\varepsilon, / \mathrm{M}^{-1} \mathrm{~cm}^{-1}\right) 461.0$ (15380), 308.0 inf. (4530) 286 (6820). IR $(\mathrm{KBr}) \vee$ 3100, 3020, 2540, $2220(\mathrm{CN}), 1570,1540,1500,1470,1440,1420,1360$, $1350,1320,1270,1260,1230,1200,1160,1060,980,940,800,740,720,600 \mathrm{~cm}^{-1} .{ }^{1} \mathrm{H}$ $\operatorname{NMR}\left(\mathrm{CDCl}_{3}\right) \delta 3.96\left(\mathrm{~s}, 3 \mathrm{H}, \mathrm{OCH}_{3}\right) 6.23\left(\mathrm{~d}, 1 \mathrm{H}, J=4.1 \mathrm{~Hz}, 4^{\prime}-\mathrm{H}\right), 7.08(\mathrm{~d}, 1 \mathrm{H}, J=4.1$ $\left.\mathrm{Hz}, 3^{\prime}-\mathrm{H}\right), 7.15$ (d, $\left.1 \mathrm{H}, J=4.1,3-\mathrm{H}\right), 7.57$ (d, $\left.1 \mathrm{H}, J=4.1 \mathrm{~Hz}, 4-\mathrm{H}\right), 7.71$ (s, $1 \mathrm{H}$, $\left.\mathrm{CH}=\mathrm{C}(\mathrm{CN})_{2}\right) .{ }^{13} \mathrm{C} \mathrm{NMR}\left(\mathrm{CDCl}_{3}\right) \delta 60.47,105.74,113.70,114.55,121.44,122.65$, 126.31, 132.09, 140.53, 149.94, 150.78, 169.41. MS (EI) m/z (\%): $272\left(\mathrm{M}^{+}, 70\right), 229$ (18), 224 (7), 203 (9), 185 (16), 149 (4), 121 (3), 69 (9). HRMS: m/z (EI) for $\mathrm{C}_{13} \mathrm{H}_{8} \mathrm{~N}_{2} \mathrm{OS}_{2}$; calcd 272.0078; found: 272.0077 .

5-Dicyanovinyl-5'-ethoxy-2,2'-bithiophene 5b : dark orange solid (48\%). Mp: 159-161 ${ }^{\circ} \mathrm{C}$ (ether). UV (EtOH): $\lambda_{\max } \mathrm{nm}\left(\varepsilon, / \mathrm{M}^{-1} \mathrm{~cm}^{-1}\right) 462.0$ (14897), $307.0 \mathrm{sh}$ (4520), 286.0 (7185). IR (KBr) v 2940, 2860, $2220(\mathrm{CN}), 1570,1540,1490,1460,1400,1360,1320$, $1260,1200,1160,1110,1025,940,880,800,785,730,640 \mathrm{~cm}^{-1} .{ }^{1} \mathrm{H} \mathrm{NMR}\left(\mathrm{CDCl}_{3}\right)$ $\delta 1.45\left(\mathrm{t}, 3 \mathrm{H}, J=7.2 \mathrm{~Hz}, \mathrm{OCH}_{2} \mathrm{CH}_{3}\right), 4.16\left(\mathrm{q}, 2 \mathrm{H}, J=7.2 \mathrm{~Hz}, \mathrm{OCH}_{2} \mathrm{CH}_{3}\right), 6.22(\mathrm{~d}, 1 \mathrm{H}$, $\left.J=4.2 \mathrm{~Hz}, 4^{\prime}-\mathrm{H}\right), 7.07$ (d, $\left.1 \mathrm{H}, J=4.2 \mathrm{~Hz}, 3^{\prime}-\mathrm{H}\right), 7.15$ (d, $\left.1 \mathrm{H}, J=4.0 \mathrm{~Hz}, 3-\mathrm{H}\right), 7.56$ (d, 1 $\mathrm{H}, J=4.0 \mathrm{~Hz}, 4-\mathrm{H}), 7.70\left(\mathrm{~s}, 1 \mathrm{H}, \mathrm{CH}=\mathrm{C}(\mathrm{CN})_{2}\right) .{ }^{13} \mathrm{H} \mathrm{NMR} \delta\left(\mathrm{CDCl}_{3}\right) 14.59,69.87$, $106.51,113.73,114.58,121.32,122.59,126.38,132.03,140.50,149.89,150.95$, 168.52. MS (EI) m/z (\%): $286\left(\mathrm{M}^{+}, 75\right), 258$ (76), 257 (100), 229 (22), 219 (8), 203 (13), 185 (14), 111 (6), 83 (9), 69 (23), 57 (14). HRMS: m/z (EI) for $\mathrm{C}_{14} \mathrm{H}_{10} \mathrm{~N}_{2} \mathrm{OS}_{2}$; calcd 286.0234; found: 286.0230.

5-Dicyanovinyl-5'-N,N-dimethylamino-2,2'-bithiophene 5 $\mathbf{d}^{3}$ : violet solid (88\%). Mp: 223-225 ${ }^{\circ} \mathrm{C}$ (ether) [lit $\left.{ }^{3} .241-242{ }^{\circ} \mathrm{C}\right]$. UV (EtOH): $\lambda \max \mathrm{nm}\left(\varepsilon, / \mathrm{M}^{-1} \mathrm{~cm}^{-1}\right) 560.0$ (20842), 356.0 inf. (4370), 337 (5680), 303.0 (4630). IR (KBr) v 3120, 2215 (CN) $1570,1500,1470,1440,1420,1400,1340,1280,1235,1200,1160,1140,1060,920$, 900, 780, 750, 680, $640 \mathrm{~cm}^{-1} .{ }^{1} \mathrm{H} \mathrm{NMR}\left(\mathrm{CDCl}_{3}\right) \delta 3.06\left(\mathrm{~s}, 6 \mathrm{H}, \mathrm{N}\left(\mathrm{CH}_{3}\right)_{2}\right), 5.86(\mathrm{~d}, 1 \mathrm{H}$, $\left.J=4.2 \mathrm{~Hz}, 4^{\prime}-\mathrm{H}\right), 6.95$ (d, $\left.1 \mathrm{H}, J=4.2 \mathrm{~Hz}, 3^{\prime}-\mathrm{H}\right), 7.26$ (overlapped d, 1 H, 3-H) 7.49 (d, 1 
$\mathrm{H}, J=4.2 \mathrm{~Hz}, 4-\mathrm{H}), 7.61\left(\mathrm{~s}, 1 \mathrm{H}, \mathrm{CH}=\mathrm{C}(\mathrm{CN})_{2}\right) .{ }^{13} \mathrm{C} \mathrm{NMR}\left(\mathrm{CDCl}_{3}\right) \delta 42.30,103.61$, $114.57,115.47,116.98,118.38,120.65,128.66,130.05,130.26,141.23,149.04 . \mathrm{MS}$ (EI) $m / z(\%): 285\left(\mathrm{M}^{+}, 100\right), 270$ (31), 237 (7), 229 (8), 185 (11), 143 (13), 69 (6).

5-Dicyanovinyl-5'-N,N-diethylamino-2,2'-bithiophene 5e ${ }^{29}$ : dark violet solid (51\%). Mp: $163-165^{\circ} \mathrm{C}$ (ether) [lit $\left.{ }^{29} 168-170^{\circ} \mathrm{C}\right]$. UV (EtOH): $\lambda \max n$ (14684), 357.0 inf. (5680), 340.0 (6650), 306.0 (5060), 270.0 (4895), 247.0 (5980). IR $(\mathrm{KBr}) \vee$ 2960, 2920, $2215(\mathrm{CN}), 1570,1510,1360,1340,1270,1140,1080,1060$, 1000, 930, 880, 860, 780, $740 \mathrm{~cm}^{-1}$. ${ }^{1} \mathrm{H} \mathrm{NMR}\left(\mathrm{CDCl}_{3}\right) \delta 1.23(\mathrm{t}, 6 \mathrm{H}, J=7.1 \mathrm{~Hz}$, $2 \mathrm{xCH}_{2} \mathrm{CH}_{3}$ ), 3.31 (q, $\left.4 \mathrm{H}, J=7.1 \mathrm{~Hz}, 2 \mathrm{xCH}_{2} \mathrm{CH}_{3}\right), 5.86$ (d, $\left.1 \mathrm{H}, J=4.3 \mathrm{~Hz}, 4^{\prime}-\mathrm{H}\right), 6.91$ (d, $1 \mathrm{H}, J=4.3 \mathrm{~Hz}, 3^{\prime}-\mathrm{H}$ ), 7.25 (overlapped d, $1 \mathrm{H}, 3-\mathrm{H}$ ), 7.46 (d, $1 \mathrm{H}, J=4.3 \mathrm{~Hz}, 4-\mathrm{H}$ ), 7.56 (s, $\left.1 \mathrm{H}, \mathrm{CH}=\mathrm{C}(\mathrm{CN})_{2}\right) .{ }^{13} \mathrm{C} \mathrm{NMR}\left(\mathrm{CDCl}_{3}\right) \delta 47.49,102.92,114.79,115.68,116.88$, 120.26, 129.85, 130.42, 141.36, 148.75, 152.88, 161.17. MS (EI) m/z (\%): $313\left(\mathrm{M}^{+}\right.$, 100), 298 (86), 284 (13), 269 (23), 256 (13), 242 (7), 230 (5), 197 (9), 158 (8), 141 (3), 96 (15), 69 (4). HRMS: $m / z$ (EI) for $\mathrm{C}_{16} \mathrm{H}_{15} \mathrm{~N}_{3} \mathrm{~S}_{2}$; calcd 313.0707; found: 313.0708.

5-Dicyanovinyl-5' $-N, N$-diisopropylamino-2,2'-bithiophene 5f: violet solid (45\%). Mp: 154.5-156 ${ }^{\circ} \mathrm{C}$ (ether). UV (EtOH): $\lambda_{\max } \mathrm{nm}\left(\varepsilon, / \mathrm{M}^{-1} \mathrm{~cm}^{-1}\right) 580.0$ (20920), 343.0 (12564), 314.0 inf. (7860). IR (KBr) v 2930, 2850, 2220 (CN), 1580, 1560, 1530, 1480, 1440, 1400, 1350, 1330, 1280, 1230, 1160, 1140, 1100, 1060, 920, 820, 780, 760, 660, $640 \mathrm{~cm}^{-1} .{ }^{1} \mathrm{H} \mathrm{NMR}\left(\mathrm{CDCl}_{3}\right) \delta 1.31\left(\mathrm{~d}, 12 \mathrm{H}, J=6.7 \mathrm{~Hz}, 2 \mathrm{xCH}\left(\mathrm{CH}_{3}\right)_{2}\right), 3.84(\mathrm{sep}, 2 \mathrm{H}$, $\left.J=6.7 \mathrm{~Hz}, 2 \mathrm{xCH}\left(\mathrm{CH}_{3}\right)_{2}\right), 6.02\left(\mathrm{~d}, 1 \mathrm{H}, J=4.3 \mathrm{~Hz}, 4^{\prime}-\mathrm{H}\right), 6.93\left(\mathrm{~d}, 1 \mathrm{H}, J=4.3 \mathrm{~Hz}, 3^{\prime}-\mathrm{H}\right)$, 7.26 (overlapped d, $1 \mathrm{H}, 3-\mathrm{H}), 7.47$ (d, $1 \mathrm{H}, \mathrm{J}=3.8 \mathrm{~Hz}, 4-\mathrm{H}), 7.58\left(\mathrm{~s}, 1 \mathrm{H}, \mathrm{CH}=\mathrm{C}(\mathrm{CN})_{2}\right)$. ${ }^{13} \mathrm{C} \mathrm{NMR} \delta\left(\mathrm{CDCl}_{3}\right) \delta 20.19,51.73,106.23,114.78,115.68,117.09,120.40,129.62$, 130.01, 141.26, 148.81, 152.78, 159.46. MS (EI) $\mathrm{m} / \mathrm{z}(\%): 341\left(\mathrm{M}^{+}, 100\right), 326(46), 298$ (16), 293 (17), 284 (50), 256 (64), 236 (7), 197 (10), 149 (13), 139 (6), 111 (6), 96 (23), 83 (22), 69 (15). HRMS: $m / z$ (EI) for $\mathrm{C}_{18} \mathrm{H}_{19} \mathrm{~N}_{3} \mathrm{~S}_{2}$; calcd 341.1020 found: 341.1023.

5-Dicyanovinyl-5'-piperidino-2,2'-bithiophene 5g': dark violet solid (81\%). Mp: 171$172.5{ }^{\circ} \mathrm{C}$ (ether) [lit $\left.{ }^{4} .169-172{ }^{\circ} \mathrm{C}\right] . \mathrm{UV}(\mathrm{EtOH}): \lambda_{\max } \mathrm{nm}\left(\varepsilon, / \mathrm{M}^{-1} \mathrm{~cm}^{-1}\right) 564.0$ (35268), 357.0 inf.(4762), 336.0 (7222), 302.0 (6713), 246.0 (6759), 211.0 (7926),.343.0 (18039), 241.5 (4914), 214.0 (6102). IR (KBr) v 2940, $2215(\mathrm{CN}), 1570,1490,1470$, 1430, 1380, 1330, 1255, 1250, 1180, 1130, 1125, 1120, 1080, 1010, 920, 895, 860, 800, $760,660 \mathrm{~cm}^{-1} .{ }^{1} \mathrm{H} \mathrm{NMR}\left(\mathrm{CDCl}_{3}\right) \delta 1.60-1.80\left(\mathrm{~m}, 6 \mathrm{H}, 3 \mathrm{xCH}_{2}\right), 3.25-3.30(\mathrm{~m}, 4 \mathrm{H}$, 
2xCH $\left.\mathrm{CH}_{2}\right), 6.02$ (d, $\left.1 \mathrm{H}, J=4.3 \mathrm{~Hz}, 4^{\prime}-\mathrm{H}\right), 6.96$ (d, $\left.1 \mathrm{H}, J=4.3 \mathrm{~Hz}, 3^{\prime}-\mathrm{H}\right), 7.24$ (overlapped d, $1 \mathrm{H}, 3-\mathrm{H}), 7.50(\mathrm{~d}, 1 \mathrm{H}, J=4.3 \mathrm{~Hz}, 4-\mathrm{H}), 7.62\left(\mathrm{~s}, 1 \mathrm{H}, \mathrm{CH}=\mathrm{C}(\mathrm{CN})_{2}\right) .{ }^{13} \mathrm{C} \mathrm{NMR}$ $\left(\mathrm{CDCl}_{3}\right) \delta 23.52,24.93,51.39,104.85,114.47,115.38,118.87,120.96,129.55,130.52$, 141.12, 149.10, 152.38, 163.29. MS (EI) $m / z$ (\%): 325 (M+, 100), 269 (7), 256 (5), 237 (4), 217 (4), 197 (7), 162 (10), 151 (7), 120 (4), 96 (4). HRMS: m/z (EI) for $\mathrm{C}_{17} \mathrm{H}_{15} \mathrm{~N}_{3} \mathrm{~S}_{2}$; calcd 325.0707; found: 325.0703 .

General procedure for the synthesis of 5-tricyanovinyl-2,2'-bithiophenes 6 from $2,2^{\prime}$-bithiophenes 1 by tricyanovinylation with tetracyanoethylene (TCNE)

A solution of 2,2'-bithiophenes $1(1.3 \mathrm{mmol})$ in DMF $(2 \mathrm{ml})$ was cooled at $0{ }^{\circ} \mathrm{C}$ and then TCNE $(0.128 \mathrm{~g}, 1 \mathrm{mmol})$ was added slowly. The reaction mixture was stirred overnight at room temperature. After this time the mixture was poured into ice/water and the precipitate filtered off and washed several times with water, petrol and ether. The solid obtained was purified by recristallization to give the pure 5-tricyanovinyl2,2'-bithiophenes 6.

5-Tricyanovinyl-5'-methoxy-2,2'-bithiophene 6a: violet solid (53\%). Mp: 167.5-169 ${ }^{\circ} \mathrm{C}$ (ether). UV (EtOH): $\lambda \max \mathrm{nm}\left(\varepsilon, / \mathrm{M}^{-1} \mathrm{~cm}^{-1}\right) 545.0$ (24632), 345.0 inf. (5102), 313.0 (7561), 246.0 (4061), 213.0 (4520). IR (KBr) v 3000, 2980, 2225 (CN), 1520, 1480, 1420, 1320, 1260, 1160, 1100, 1060, 970, 800, 780, 740, 700, $640 \mathrm{~cm}^{-1}$. ${ }^{1} \mathrm{H}$ NMR $\left(\mathrm{DMSO}_{-} \mathrm{d}_{6}\right) \delta 3.99\left(\mathrm{~s}, 3 \mathrm{H}, \mathrm{OCH}_{3}\right) 6.55\left(\mathrm{~d}, 1 \mathrm{H}, J=4.2 \mathrm{~Hz}, 4^{\prime}-\mathrm{H}\right), 7.57$ (d, $1 \mathrm{H}, J=4.2$ $\left.\mathrm{Hz}, 3^{\prime}-\mathrm{H}\right), 7.65$ (d, $\left.1 \mathrm{H}, J=4.2 \mathrm{~Hz}, 3-\mathrm{H}\right), 8.03$ (d, $\left.1 \mathrm{H}, J=4.2 \mathrm{~Hz}, 4-\mathrm{H}\right) .{ }^{13} \mathrm{C}$ NMR $\left(\right.$ DMSO-d $\left._{6}\right) \delta 61.15,107.61,113.20,113.31,113.41,120.07,124.75,130.05,130.26$, 130.79, 142.62, 152.19, 170.72. MS (EI) m/z (\%): 297 (M+, 66), 282 (100), 254 (12), 228 (9), 210 (7), 183 (5), 69 (10). HRMS: $m / z$ (EI) for $\mathrm{C}_{14} \mathrm{H}_{7} \mathrm{~N}_{3} \mathrm{OS}_{2}$; calcd 297.0031; found: 297.0036.

5-Tricyanovinyl-5'-ethoxy-2,2'-bithiophene 6b: violet solid (70\%). Mp: 179-181 ${ }^{\circ} \mathrm{C}$ (ether). UV (EtOH): $\lambda \max \mathrm{nm}\left(\varepsilon, / \mathrm{M}^{-1} \mathrm{~cm}^{-1}\right) 549.0$ (26451), 338.0 inf. (6536), 315.0 (8304), 245.0 (4627), 212.0 (5823). IR (KBr) v 3000, 2980, 2220 (CN), 1550, 1520, 1480, 1460, 1420, 1380, 1360, 1310, 1260, 1180, 1100, 1060, 1020, 880, 860, 800, 780, $770,760 \mathrm{~cm}^{-1}$. ${ }^{1} \mathrm{H}$ NMR $\delta\left(\mathrm{DMSO}_{6}\right) 1.37\left(\mathrm{t}, 3 \mathrm{H}, J=7.2 \mathrm{~Hz}, \mathrm{OCH}_{2} \mathrm{CH}_{3}\right), 4.25(\mathrm{q}, 2$ $\left.\mathrm{H}, J=7.2 \mathrm{~Hz}, \mathrm{OCH}_{2} \mathrm{CH}_{3}\right), 6.54$ (d, $\left.1 \mathrm{H}, J=4.2 \mathrm{~Hz}, 4^{\prime}-\mathrm{H}\right), 7.56\left(\mathrm{~d}, 1 \mathrm{H}, J=4.2 \mathrm{~Hz}, 3^{\prime}-\mathrm{H}\right)$, 
7.65 (d, $1 \mathrm{H}, J=4.2 \mathrm{~Hz}, 3-\mathrm{H}$ ), 8.02 (d, $1 \mathrm{H}, J=4.2 \mathrm{~Hz}, 4-\mathrm{H}$ ). ${ }^{13} \mathrm{C}$ NMR (DMSO-d 6 ) $\delta$ 14.36, 70.27, 108.15, 113.20, 113.29, 113.41, 119.95, 124.70, 130.16, 130.20, 130.68, 142.63, 152.31, 169.77. MS (EI) m/z (\%): $311\left(\mathrm{M}^{+}, 50\right), 283$ (100), 282 (53), 254 (11), 228 (10), 210 (13), 181 (13), 153 (7), 127 (4), 111 (6), 69 (9). HRMS: m/z (EI) for $\mathrm{C}_{15} \mathrm{H}_{9} \mathrm{~N}_{3} \mathrm{OS}_{2}$; calcd 311.0187; found: 311.0188 .

5-Tricyanovinyl-5'- $N, N$-dimethylamino-2,2'-bithiophene $\mathbf{6 d}^{5,29}$ : dark blue solid (82\%). Mp: $273^{\circ} \mathrm{C}$ (ether) [lit $\left.{ }^{5} .275^{\circ} \mathrm{C}\right]$. UV (EtOH): $\lambda \max \mathrm{nm}\left(\varepsilon, / \mathrm{M}^{-1} \mathrm{~cm}^{-1}\right) 588.0$ (10000), 388.0 (6270), 345.0 (7620), 206.0 (12330). IR (KBr) v 3080, 2920, 2200 (CN) 1550, 1500, 1440, 1390, 1360, 1340, 1290, 1260, 1240, 1200, 1080, 1060, 910, 780, 750, 680, $640 \mathrm{~cm}^{-1} .{ }^{1} \mathrm{H}$ NMR (DMSO-d 6 ) $\delta 3.24\left(\mathrm{~s}, 6 \mathrm{H}, 2 \mathrm{xCH}_{3}\right), 6.45\left(\mathrm{~d}, 1 \mathrm{H}, J=4.3 \mathrm{~Hz}, 4^{\prime}-\mathrm{H}\right)$, 7.44 (d, $\left.1 \mathrm{H}, J=4.3 \mathrm{~Hz}, 3^{\prime}-\mathrm{H}\right), 7.85$ (d, $\left.1 \mathrm{H}, J=4.3 \mathrm{~Hz}, 3-\mathrm{H}\right), 7.89$ (d, $1 \mathrm{H}, J=4.3 \mathrm{~Hz}, 4-$ H). MS (EI) $m / z(\%): 310\left(\mathrm{M}^{+}, 8\right), 273$ (100), 272 (75), 258 (26), 257 (20), 247 (20), 240 (9), 229 (7), 224 (10), 209 (16), 194 (7), 172 (7), 146 (5), 137 (10), 96 (6), 69 (7).

5-Tricyanovinyl-5'- $N, N$-diethylamino-2,2'-bithiophene $\mathbf{6 e}^{29}$ : dark blue solid $(51 \%)$. Mp: $255-257^{\circ} \mathrm{C}$ (ether) [lit. ${ }^{4} 264-265^{\circ} \mathrm{C}$ ]. UV (EtOH): $\lambda \max$ nm 592.0 (38425), 485.0 (9200), 396.0 (1040), 379.0 (10125), 349.0 (11375), 248.0 (8075), 213.0 (10375). IR $(\mathrm{KBr}) \vee 2924,2182(\mathrm{CN}), 1550,1550,1503,1411,1334,1289,1225,1137,1103$, 1069, 1054, 1069, 1059, 997, 863, 788, 663, $642 \mathrm{~cm}^{-1} .{ }^{1} \mathrm{H}$ NMR (DMSO-d 6 ) $\delta 1.20$ (t, $6 \mathrm{H}, J=7.0 \mathrm{~Hz}, 2 \mathrm{xCH}_{2} \mathrm{CH}_{3}$ ), 3.50 (q, $\left.4 \mathrm{H}, J=7.0 \mathrm{~Hz}, 2 \mathrm{xCH}_{2} \mathrm{CH}_{3}\right), 6.48$ (d, $1 \mathrm{H}, J=4.2$ $\left.\mathrm{Hz}, 4^{\prime}-\mathrm{H}\right), 7.40$ (d, $\left.1 \mathrm{H}, J=4.2 \mathrm{~Hz}, 3^{\prime}-\mathrm{H}\right), 7.78$ (d, $\left.1 \mathrm{H}, J=4.2 \mathrm{~Hz}, 3-\mathrm{H}\right), 7.89$ (d, $1 \mathrm{H}$, $J=4.3 \mathrm{~Hz}, 4-\mathrm{H}$ ). MS (EI) $\mathrm{m} / \mathrm{z}$ (\%) 338 (M+, 28), 323 (30), 301 (93), 286 (100), 272 (15), 256 (22), 237 (13), 217 (16), 184 (5), 146 (5), 96 (11), 73 (11). HRMS: m/z (EI) for $\mathrm{C}_{17} \mathrm{H}_{14} \mathrm{~N}_{4} \mathrm{~S}_{2}$; calcd 338.0660; found: 338.0668 .

5-Tricyanovinyl-5'- $N, N$-diisopropylamino-2,2'-bithiophene 6f: dark violet solid (87\%). Mp: 197-199 ${ }^{\circ} \mathrm{C}$ (ether). UV (EtOH): $\lambda_{\max } \mathrm{nm}\left(\varepsilon, / \mathrm{M}^{-1} \mathrm{~cm}^{-1}\right) 623.0$ (13580), $595.0 \mathrm{inf}$. (12050), 496.0 (6870), 411.0 (10920), 395.0 (10970), 359.0 (11661), 261.0 (3580). IR $(\mathrm{KBr}) \vee$ 2924, $2195(\mathrm{CN}), 1539,1354,1214,1118,915,786,720,665,632 \mathrm{~cm}^{-1} .{ }^{1} \mathrm{H}$ NMR (DMSO-d 6 ) $\delta 1.28\left(\mathrm{~d}, 12 \mathrm{H}, J=6.6 \mathrm{~Hz}, 2 \times \mathrm{CH}\left(\mathrm{CH}_{3}\right)_{2}\right), 3.90-4.00(\mathrm{~m}, 2 \mathrm{H}$, 2xCH( $\left.\left.\mathrm{CH}_{3}\right)_{2}\right), 6.63\left(\mathrm{~d}, 1 \mathrm{H}, J=4.5 \mathrm{~Hz}, 4^{\prime}-\mathrm{H}\right), 7.48\left(\mathrm{~d}, 1 \mathrm{H}, J=4.5 \mathrm{~Hz}, 3^{\prime}-\mathrm{H}\right), 7.78(\mathrm{~d}, 1$ H, J=4.5 Hz, 3-H), 7.94 (d, $1 \mathrm{H}, J=4.5 \mathrm{~Hz}, 4-\mathrm{H})$. MS (EI) m/z (\%): $366\left(\mathrm{M}^{+}, 60\right), 361$ (27), 351 (30), 343 (16), 329 (89), 318 (39), 309 (45), 303 (22), 286 (30), 272 (55), 265 
(51), 244 (100) 234 (10), 222 (20), 208 (20), 180 (45), 146 (8), 121 (7), 106 (8), 96 (46), 86 (51). HRMS: $m / z$ (EI) for $\mathrm{C}_{18} \mathrm{H}_{19} \mathrm{~N}_{3} \mathrm{~S}_{2}$; calcd 366.0976, found: 366.0973 .

5-Tricyanovinyl-5'-piperidino-2,2'-bithiophene 6g ${ }^{35}$ : dark violet solid (81\%). Mp: 241$243{ }^{\circ} \mathrm{C}$ (ether). UV (EtOH): $\lambda_{\max } \mathrm{nm}\left(\varepsilon, / \mathrm{M}^{-1} \mathrm{~cm}^{-1}\right) 591.0$ (23560), 472.0 (20320), 397.0 (21000), 338.0 (21740), 220.0 (27000). IR (KBr) v 3080, 2940, 2860, 2200 (CN), 1540, 1500, 1470, 1450, 1400, 1390, 1360, 1340, 1280, 1240, 1180, 1100, 1070, 1010, $900,880,850,830,780,760,640 \mathrm{~cm}^{-1} .{ }^{1} \mathrm{H}$ NMR $\delta_{\mathrm{H}}\left(\mathrm{DMSO}-\mathrm{d}_{6}\right) 1.40-1.80(\mathrm{~m}, 6 \mathrm{H}$, $\left.3 \times \mathrm{XH}_{2}\right), 3.15-3.30\left(\mathrm{~m}, 4 \mathrm{H}, 2 \mathrm{xCH}_{2}\right), 6.65\left(\mathrm{~d}, 1 \mathrm{H}, J=4.8 \mathrm{~Hz}, 4^{\prime}-\mathrm{H}\right), 7.48$ (d, $1 \mathrm{H}, J=4.8$ Hz, 3'-H), 7.81 (d, 1 H, J=4.8 Hz, 3-H), 7.95 (d, 1 H, $J=4.8 \mathrm{~Hz}, 4-\mathrm{H})$. MS (EI) $m / z$ (\%): 350 ( $\left.\mathrm{M}^{+}, 17\right), 313$ (100), 312 (47), 287 (9), 217 (4), 249 (10), 73 (6). HRMS: m/z (EI) for $\mathrm{C}_{18} \mathrm{H}_{14} \mathrm{~N}_{4} \mathrm{~S}_{2}$; calcd 350.065349; found: 350.065990 .

5-Tricyanovinyl-5'-(4-methoxyanilino)-2,2'-bithiophene 6h: dark violet solid (69\%). Mp: $282-284{ }^{\circ} \mathrm{C}$ (ether). UV (EtOH): $\lambda \max \mathrm{nm}\left(\varepsilon, / \mathrm{M}^{-1} \mathrm{~cm}^{-1}\right) 688.0$ (11000), 603.0 (8400), 565.0 (8400), 221.0 (11000). IR (liquid film) v $3583(\mathrm{NH}), 2213(\mathrm{CN}), 1605$, 1502, 1480, 1408, 1384, 1303, 1253, 1156, 1116, 1092, 1092, 1029, 834, 789, 666 $\mathrm{cm}^{-1} .{ }^{1} \mathrm{H}$ NMR $\delta$ (DMSO-d $) 3.90$ (s, $\left.3 \mathrm{H}, \mathrm{OCH}_{3}\right), 7.04$ (d, $\left.1 \mathrm{H}, J=5.0 \mathrm{~Hz}, 4^{\prime}-\mathrm{H}\right), 7.23$ (m, $3 \mathrm{H}, 3^{\prime}-\mathrm{H}$ and 2xAr-H), 7.41 (d, $\left.2 \mathrm{H}, J=9.0 \mathrm{~Hz}, 2 \mathrm{xAr}-H\right), 7.89$ (d, $1 \mathrm{H}, J=5.0 \mathrm{~Hz}, 3-$ H), 7.95 (d, $1 \mathrm{H}, J=5.0 \mathrm{~Hz}, 4-\mathrm{H})$. MS (EI) m/z (\%): 388 (M+, 99), 387 (100), 373 (54), 349 (47), 334, (30), 231 (12), 219 (5), 181 (7), 165 (16), 123 (54), 108 (68), 92 (20). HRMS: $m / z$ (EI) for $\mathrm{C}_{20} \mathrm{H}_{12} \mathrm{~N}_{4} \mathrm{OS}_{2}$; calcd 388.0452; found: 388.0437 .

\section{References}

1. Effenberger F.; Wuerthner F., Angew. Chem. 1993, 105 , 742-744.

2. Effenberger F.; Wuerthner F., Angew. Chem. Int. Ed. Eng. 1993, 32, 719-721.

3. Effenberger F.; Wuerthner F.; Steybe F., J. Org. Chem. 1995, 60, 2082-2091.

4. Bedworth P. V.; Cai Y.; Jen A.; Marder S. R., J. Org. Chem. 1996, 61, 2242-2246.

5. Steybe F.; Effenberger F; Beckman S.; Kramer P.; Glania C.; Wortmann R., Chem. Phys. 1997, 219, 317-331.

6. Bauerle P. In: The Synthesis of Oligothiophenes, D. Fichou Ed. Handbook of Oligoand Polythiophenes, Wiley-VCH: Weinheim, 1999; Cap. 3, pp 89-173. 
7. Mignani G.; Leising F.; Meyrueix R.; Samson M., Tetrahedron Lett. 1990, 31 (33), 4743-4746.

8. Hutchins M. G.; Ferguson I.; McGeein D. J.; Morley J. O.; Ziss J.; Ledoux I., J. Chem. Soc. Perkin Trans 2 1995, 171- 176.

9. Steybe F.; Effenberger F; Gubler U.; Bosshard C.; Gunter P., Tetrahedron 1998, $54,8469-8480$.

10. Wuerthner F.; Effenberger F.,Chem. Phys. 1993, 173, 305-314.

11. Zyss D. S. In: Non linear optical properties of organic molecules and crystals, Vol 1 and 2; Academic Press: Orlando, 1987.

12. Brosshard C.; Sutter K.; Petre P.; Hulliger J.; Florsheimer M.; Kaatz M.; Gunter P. In: Organic non-linear optical materials, Gordon and Breach Science Publishers, Amsterdam, 1995.

13. Prim D.; Kirsch G., J. Chem. Soc., Perkin Trans. 1 1994, 2603-2606.

14. Prim D.; Kirsch G; Leising F.; Mignani G., J. Heterocycl. Chem. 1994, 31, 1005 1009.

15. Prim D.; Joseph D.; Kirsch G., Phosphorus, Sulfur and Silicon 1994, 91, 137-143.

16. Costa S.P.G.; Griffiths; Kirsch G., Oliveira-Campos A. M. F., Anales de Quimica Int. Ed. 1998, 94, 186-188.

17. Raposo M. Manuela M.; Kirsch G., Heterocycles 2001, 55 (8), 1487-1498.

18. Lescot E., Buu-Hoi Ng. Ph.; Xuong N. D., J. Chem. Soc. 1959, 3234-3237.

19. Meth-Cohn O.; Ashton M., Tetrahedron Lett. 2000, 41, 2749-2752.

20. Raimundo J-M.; Blanchard P.; Frère P.; Mercier N.; Ledoux-Rak I.; Hierle R.; Roncali J., Tetrahedron Lett. 2001, 42, 1507-1510.

21. Raimundo J-M.; Blanchard P.; Gallego-Planas N.; Frère P.; Mercier N.; LedouxRak I.; Hierle R.; Roncali J., J. Org. Chem. 2002, 67, 205-218.

22. Parakka J. P.; Cava M. P., Tetrahedron 1995, 51 (8), 2229-2242.

23. Kromer J.; Bauerle P., Tetrahedron 2001, 57, 3785-3794.

24. Wei Y. ; Wang B.; Wang W.; Tian J., Tetrahedron Lett. 1995, 36 (5), 665-668.

25. Kim D. S. H. L.; Ashendel C. L.; Zhou Q.; Chang C.; Lee E-S.; Chang C., Bioorg. Med. Chem. Lett. 1998, 8, 2295-2698.

26. Chan H. S. O.; Choon S., Prog. Polym. Sci. 1998, 23, 1167-1231.

27. Blockhuys F.; Hoefnagels R.; Peten C.; Alsenoy C. V.; Geise H. J., Journal of Molecular Structure 1999, 485-486, 87-96. 
28. Tietze L. F. In: The Knoevenagel Reaction, Trost B. M. Ed. Comprehensive Organic Synthesis, Pergamond Press: Oxford, 1991; Coll Vol. 2, pp 358-359.

29. Eckert K.; Schroder A.; Hartmann H., Eur. J. Org. Chem 2000, 1327-1334.

30. McKusick B.C.; Heckert, R. E.; Cairns T. L.; Coffmann D. D.; Mower H. F., J. Am. Chem. Soc., 1958, 80, 2806-2815.

31. Rao V. P.; Jen A. K-Y.; Wong K. Y.; Drost K. J., J. Chem. Soc. Chem. Commun., 1993, 14, 1118-1120.

32. Bu X. R.; Li H.; Derver D. V.; Mintz E. A., Tetrahedron Lett. 1996, 367 (41), 7331-7334.

33. Cai C.; Liakatas I.; Wong M-S.; Bosh M.; Bosshard C.; Gunter P.; Concilio S.; Tirelli N.; Suter U. W., Org. Lett. 1999, 1 (11), 1847-1849.

34. Fatiadi A. J., Synthesis 1986, 249.

35. Jen K-Y. A.; Rao V. P.; Drost K. J., Cai Y.; Mininni R. M.; Kenney J. T.; Binkley E. S.; Dalton L. R., Proc. SPIE-Int. Soc. Opt. Eng. 1994, 2285 (Chem. Abstr., 1995, $123,84708 f)$.

\section{Acknowledgements}

Thanks are due to ICCTI/French Embassy (Technical and Scientific Cooperation Programme) and to FCT for financial support through IBQF (UM) and POCTI (ref. POCTI/QUI/37816/2001) as well as for a sabbatical grant to M. M. M. Raposo (FMRH / BSAB / 134/99). 


\section{Captions}

Table 1. Synthesis of formyl-derivatives $\mathbf{2 f - g}$ and $\mathbf{3 f - g}$ from bithiophenes $\mathbf{1 f}-\mathbf{g}$ by Vilsmeier-Haack reaction.

Table 2. Synthesis of 5-formyl- derivatives $\mathbf{4}$ from bithiophenes $\mathbf{1}$ by lithiation followed by reaction with DMF.

Table 3. Synthesis of 5-dicyanovinylbithiophenes 5 from 5-formylbithiophenes 4 by Knoevenagel condensation with malononitrile.

Table 4. Synthesis of 5-tricyanovinylbithiophenes 6 from bithiophenes $\mathbf{1}$ by tricyanovinylation reaction with TCNE. 
TABLE 1

\begin{tabular}{ccccc}
\hline Compound & $\mathbf{R}$ & Yield (\%) & IR $v_{\mathbf{C H O}}\left[\mathbf{c m}^{-1}\right]$ & $\begin{array}{c}\text { UV/Vis. (Ethanol) } \\
\lambda_{\text {max }}[\mathbf{n m}](\varepsilon)\end{array}$ \\
\hline $\mathbf{2 f}$ & $\mathrm{N}(\mathrm{Pr}-i)_{2}$ & 41 & 1668 & $313.0(10878)$ \\
$\mathbf{3 f}$ & $\mathrm{N}(\mathrm{Pr}-i)_{2}$ & 10 & 1665 (broad) & $343.0(18039)$ \\
$\mathbf{2 g}$ & piperidino & 80 & 1660 & $332.0(14794)$ \\
$\mathbf{3 g}$ & piperidino & 3 & 1659,1650 & $397.0(14735)$ \\
\hline
\end{tabular}

TABLE 2

\begin{tabular}{|c|c|c|c|c|}
\hline Compound & $\mathbf{R}$ & Yield (\%) & $\operatorname{IR} v_{\mathrm{CHO}}\left[\mathrm{cm}^{-1}\right]$ & $\begin{array}{c}\text { UV/Vis. (Ethanol) } \\
\lambda_{\max }[\mathrm{nm}](\varepsilon)\end{array}$ \\
\hline $4 a$ & $\mathrm{OMe}$ & 45 & 1646 & $385.0(22351)$ \\
\hline $4 b$ & OEt & 56 & 1660 & 383.0 (15579) \\
\hline $4 c$ & OPr $-i$ & 27 & 1652 & 386.5 (17133) \\
\hline $4 d$ & $\mathrm{NMe}_{2}$ & 86 & 1645 & $451.0(25114)$ \\
\hline $4 e$ & $\mathrm{NEt}_{2}$ & 86 & 1650 & $463.0(27107)$ \\
\hline $4 f$ & $\mathrm{~N}(\operatorname{Pr}-i)_{2}$ & 88 & 1650 & $466.0(22517)$ \\
\hline $4 g$ & piperidino & 84 & 1650 & $442.0(22133)$ \\
\hline $4 h$ & 4-methoxyanilino & 16 & 1630 & $462.0(18895)$ \\
\hline
\end{tabular}


TABLE 3

\begin{tabular}{ccccc}
\hline Compound & $\mathbf{R}$ & Yield (\%) & $\mathbf{I R} \boldsymbol{v}_{\mathbf{C N}}\left[\mathbf{c m}^{-1}\right]$ & $\begin{array}{c}\text { UV/Vis. (Ethanol) } \\
\lambda_{\mathbf{m a x}}[\mathbf{n m}](\varepsilon)\end{array}$ \\
\hline $\mathbf{5 a}$ & $\mathrm{OMe}$ & 46 & 2220 & $461.0(15380)$ \\
$\mathbf{5 b}$ & $\mathrm{OEt}$ & 48 & 2220 & $462.0(14897)$ \\
$\mathbf{5 d}$ & $\mathrm{NMe}_{2}$ & 88 & 2215 & $560.0(20842)$ \\
$\mathbf{5 e}$ & $\mathrm{NEt}_{2}$ & 51 & 2215 & $577.0(14684)$ \\
$\mathbf{5 f}$ & $\mathrm{N}(\mathrm{Pr}-i)_{2}$ & 45 & 2220 & $580.0(20920)$ \\
$\mathbf{5 g}$ & piperidino & 81 & 2215 & $564.0(35268)$ \\
\hline
\end{tabular}

TABLE 4

\begin{tabular}{ccccc}
\hline Compound & $\mathbf{R}$ & Yield (\%) & $\mathbf{I R}_{\mathbf{C N}}\left[\mathbf{c m}^{-1}\right]$ & $\begin{array}{c}\text { UV/Vis. (Ethanol) } \\
\lambda_{\mathbf{m a x}}[\mathbf{n m}](\varepsilon)\end{array}$ \\
\hline $\mathbf{6 a}$ & $\mathrm{OMe}$ & 53 & 2225 & $545.0(24632)$ \\
$\mathbf{6 b}$ & $\mathrm{OEt}$ & 70 & 2220 & $549.0(26451)$ \\
$\mathbf{6 d}$ & $\mathrm{NMe}_{2}$ & 82 & 2200 & $588.0(10000)$ \\
$\mathbf{6 e}$ & $\mathrm{NEt}_{2}$ & 51 & 2182 & $592.0(38425)$ \\
$\mathbf{6 f}$ & $\mathrm{N}(\mathrm{Pr}-i)_{2}$ & 87 & 2195 & $623.0(13580)$ \\
$\mathbf{6 g}$ & piperidino & 81 & 2200 & $591.0(23560)$ \\
$\mathbf{6 h}$ & 4-methoxyanilino & 69 & 2213 & $688.0(11000)$ \\
\hline
\end{tabular}




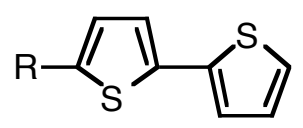

$1 f-g$

DMF- $\mathrm{POCl}_{3} / 60^{\circ} \mathrm{C}$<smiles>[R]c1sc(-c2cccs2)cc1C=O</smiles>

$2 f-g$

$3 f-g$

$$
\begin{array}{ll}
\text { f } & \mathrm{R}=\mathrm{N}(\operatorname{Pr}-\mathrm{I}) \\
\text { g } & \mathrm{R}=\text { piperidino }
\end{array}
$$

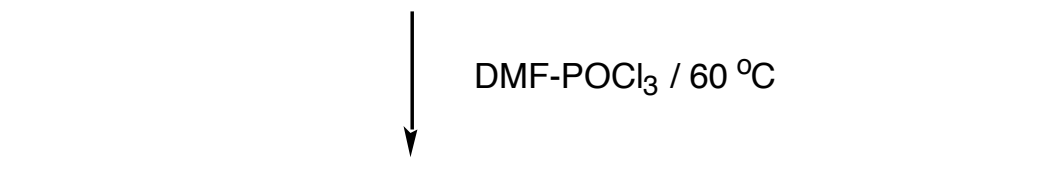

\section{Scheme 1}

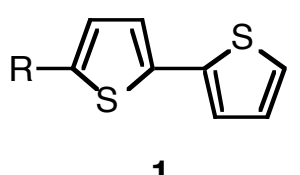

1 i, $n$-BuLi / dry ether $/ \mathrm{Ar} / 0^{\circ} \mathrm{C}$

ii, DMF / reflux.

$$
\begin{array}{ll}
\text { a } & \mathrm{R}=\mathrm{OMe} \\
\text { b } & \mathrm{R}=\mathrm{OEt} \\
\text { c } & \mathrm{R}=\mathrm{O}(\mathrm{Pr}-\mathrm{l}) \\
\text { d } & \mathrm{R}=\mathrm{NMe}_{2} \\
\text { e } & \mathrm{R}=\mathrm{NEt}_{2} \\
\text { f } & \mathrm{R}=\mathrm{N}(\mathrm{Pr}-\mathrm{l}) \\
\text { g } & \mathrm{R}=\text { piperidino } \\
\text { h } & \mathrm{R}=4 \text {-methoxyanilino }
\end{array}
$$

Scheme 2 
<smiles>[R]c1ccc(-c2ccc(C=O)s2)s1</smiles>

4

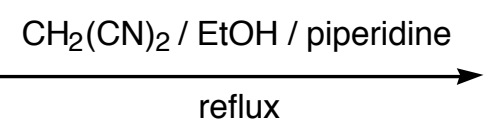

$\begin{array}{ll}\text { a } & \mathrm{R}=\mathrm{OMe} \\ \text { b } & \mathrm{R}=\mathrm{OEt} \\ \text { d } & \mathrm{R}=\mathrm{NMe}_{2} \\ \text { e } & \mathrm{R}=\mathrm{NEt}_{2} \\ \text { f } & \mathrm{R}=\mathrm{N}(\mathrm{Pr}-\mathrm{l}) \\ \text { g } & \mathrm{R}=\text { piperidino }\end{array}$

Scheme 3

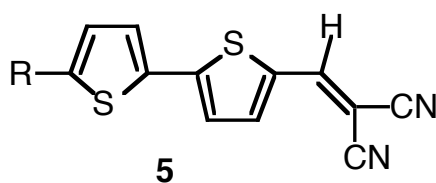

5 